\section{Diagnose Demenz: erster Bluttest auf Alzheimer in Sicht}

Nakamura A et al. High performance plasma amyloid- $\beta$ biomarkers for Alzheimer's disease. Nature 2018; 554: 249-254. doi:10.1038/nature25456

Forschern ist es gelungen, Peptide im Blut zu bestimmen, die für die AlzheimerKrankheit charakteristisch sind. Aus den Konzentrationsverhältnissen können sie überdies mit hoher Genauigkeit ablesen, ob die Blutproben von gesunden Menschen stammten, von solchen mit leichten kognitiven Störungen (MCl) oder von Alzheimer-Patienten. Dies könnte eine frühere und einfachere Diagnose der Alzheimer-Krankheit ermöglichen.

Der Test bestimmt mit hoher Genauigkeit $\beta$-Amyloid $(A \beta)$, ein Eiweißbruchstück, das sich schon Jahrzehnte vor Ausbruch der klinischen Symptome im Gehirn von Alzheimer-Patienten ansammeln kann. Es lässt sich bisher zuverlässig nur mit 2 Methoden nachweisen: mit einer Aufnahme des Gehirns mit einer speziellen Variante der Positronen-Emissions-Tomographie („Amyloid-PET“) oder mit der Entnahme von Nervenwasser im Rahmen einer Lumbalpunktion mit anschließendem Nachweis verschiedener Proteine $(A \beta$ und tau-Protein). Die erste Methode erfordert einen hohen apparativen und logistischen Aufwand mit entsprechenden Kosten, die zweite Methode kann insbesondere für ältere Patienten eine Belastung sein - einfacher wäre ein Bluttest.

\section{Massenspektroskopie statt Immunassay}

$A \beta$ findet sich im Blut aber nur in sehr geringen Konzentrationen. Versuche, es dort mit Hilfe von Immunassays (ELISA) nachzuweisen und daraus auf die Konzentrationen im Gehirn zu schließen, hatten in der Vergangenheit zu inkonsistenten Ergebnissen geführt. In der neuen Arbeit nutzten die japanischen und australischen Forscher eine Kombination aus Immunpräzipitation und Massenspektroskopie, die wesentlich empfindlicher ist als ELISA. Auch bestimmten sie nicht die Gesamtmenge an $A \beta$, sondern das
Konzentrationsverhältnis dreier $A \beta$-Varianten zueinander: $A \beta 42, \quad A \beta 40$ und APP669-711.

Getestet wurde die Zuverlässigkeit der Methode anhand zweier Gruppen von insgesamt 373 Patienten, die in Japan bzw. Australien bereits mit PET und anderen Methoden untersucht worden waren. Dabei konnte der neue Test mit hoher Zuverlässigkeit vorhersagen, ob die Studienteilnehmer Aß-Ablagerungen im Gehirn hatten oder nicht. Mit der Kombination zweier Quotienten für die verschiedenen Aß-Varianten erreichte die Vorhersagegenauigkeit $90 \%$.

\section{Anwendung in klinischen Studien}

Für die Erforschung von Therapien, die in Frühphasen der Alzheimer-Krankheit ansetzen, mit dem Ziel, den Verlauf zu verlangsamen oder ihr Fortschreiten gar zu stoppen, wäre ein verlässlicher Bluttest ein Fortschritt. Studienteilnehmer mit hoher $A \beta$-Last wären leichter zu identifizieren, und man könnte womöglich auch einfacher als bislang feststellen, welchen Einfluss Arzneikandidaten auf die Ablagerungen haben. Mittelfristig könnte ein Bluttest auch die Diagnose im Verdachtsfall verbessern oder helfen, Menschen mit hoher Belastung zu erkennen. Vor einer Zulassung müssen die Ergebnisse allerdings unabhängig bestätigt werden - und auch die Kostenfrage gilt es zu klären.

Nach einer Mitteilung der Deutschen Gesellschaft für Neurologie e.V. 